\title{
COMPACTION AND DIAGENESIS
}

\author{
A.C. FOWLER*
}

\begin{abstract}
Recent developments in the theory of compaction and diagenesis are summarised. Although these are couched in the context of the subsidence of sedimentary basins, the theory has important insights to offer in the context of groundwater flow generally, and particularly also for reactive systems. Specifically, we show how fast and slow compaction lead to normal and abnormal pore pressures, and how hysteresis in the sediment rheology can cause discontinuities in the porosity profile. We also show how 'solid density' asymptotics, or the weak solubility limit, can lead to effective reduction of complicated reaction schemes to approximate first order ones.
\end{abstract}

1. Introduction. The compaction of sedimentary basins is a problem which has practical consequences in oil-drilling operations, since the occurrence of abnormal pore pressures can lead to blow-out and collapse of the wall. Such abnormal pore pressures (i.e., above hydrostatic) can occur for a variety of reasons, and part of the purpose of modelling the system is to determine which of these are likely to be realistic causes. In this paper we will mention two, these being compaction and diagenesis.

More generally, compaction and diagenesis in sedimentary basins have mathematical connections to other systems involving reactive porous media. Two notable examples which have been studied are magma transport in the Earth's mantle (Scott and Stevenson 1984, McKenzie 1984, Fowler 1985), and reactive groundwater flow (Ortoleva et al. 1987, Dewynne et al. 1993), and there are others: as an example, the formation of biofilms on soil grains causes a reduction in porosity akin to that produced by compaction.

The situation we study is shown in Figure 1. Sediments, both organic and inorganic, are deposited at the ocean bottom and accumulate. As they do so, they compact under their weight, thus expelling pore water. If the compaction is fast (i.e., the rate of sedimentation is greater than the hydraulic conductivity of the sediments) then excess pore pressure will occur, and we quantify this here.

A second possible cause of abnormal pore pressure is clay diagenesis. This is a reaction in which smectite (a water-rich clay mineral) is transformed to illite (a water-poor clay) via the release of bound water. As it turns out, it seems that diagenesis has little quantitative impact on abnormal pore pressures (it does, though, provide a mechanism for jointing in rocks). However, its analysis is instructive because diagenesis is often thought of as occurring through precipitation and dissolution (Abercrombie et al. 1994), and the resulting model leads to a very complicated reaction system. Despite this, we illustrate how the use of the weak solubility limit (or solid density asymptotics, Ortoleva 1994) can effectively reduce

*University of Oxford, Mathematical Institute, 24-29 St. Giles', Oxford OX1 3LB England. 


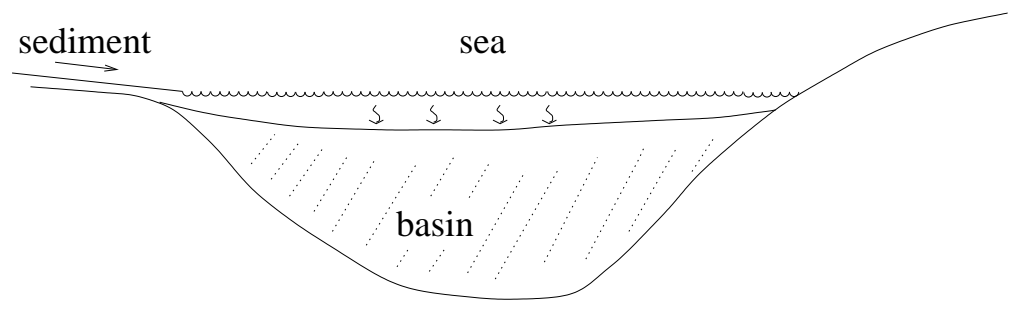

FIG. 1. Schematic of a sedimentary basin. Sediment accumulates from outflow from rivers, and also through the settlement of marine organisms.

the complicated system to first order kinetics, albeit with a complicated reaction rate.

Sedimentary basins, such as the North Sea or the Gulf of Mexico, are typically hundreds of kilometres in extent and several kilometres deep. It is thus appropriate to model the compacting system as one-dimensional, and this we do in the following section. A typical sedimentation rate is $10^{-11} \mathrm{~m}$ $\mathrm{s}^{-1}$, or $300 \mathrm{~m} \mathrm{Ma}^{-1}$, so that a 10 kilometre deep basin may accumulate in $30 \mathrm{Ma}$. On such long time scales, tectonic processes are important, and in general accumulation is not a monotonic process. If tectonic uplift occurs so that the surface of the basin rises above sea level, then erosion leads to denudation and a negative sedimentation rate. Indeed, one purpose of studying basin porosity and pore pressure profiles is to try and infer what the previous subsidence history was - an inverse problem. We shall mention one dramatic effect of such uplift on the porosity profile.

2. Compaction. The basic mathematical model is that of slow twophase flow, where the phases are solid and liquid. If the porosity is denoted by $\phi$, and the vertical coordinate is $z$, then equations of mass conservation are

$$
\begin{aligned}
\frac{\partial \phi}{\partial t}+\frac{\partial}{\partial z}\left(\phi u^{l}\right) & =0, \\
-\frac{\partial \phi}{\partial t}+\frac{\partial}{\partial z}\left[(1-\phi) u^{s}\right] & =0,
\end{aligned}
$$

where $u^{l}$ and $u^{s}$ are the liquid and solid velocities, and are related by Darcy's law

$$
\phi\left(u^{l}-u^{s}\right)=-\frac{k}{\mu}\left(\frac{\partial p}{\partial z}+\rho_{l} g\right),
$$

where $k$ is permeability, $\mu$ is viscosity of water, $p$ is pore pressure, $\rho_{l}$ is water density, and $g$ is gravity.

Two important constitutive dependences are those of the permeability and the effective pressure. The effective pressure $p_{e}$ is defined to be the difference between overburden pressure $P$ and the pore pressure: 


$$
p_{e}=P-p,
$$

and the overburden pressure is itself determined by the lithostatic gradient. In an elastic medium undergoing axial strain, matters are complicated by hoop stresses, which means that the overburden pressure is not the same as the overburden normal stress, but the difference is one of detail, and is discussed by Fowler and Yang (1998).

The effective pressure is related, in an elastic medium, to the porosity by a function $p_{e}=p_{e}(\phi)$. In a soil, the relation is hysteretic, and if allowance is made for pressure solution at depths greater than a kilometre, then an effective viscous relationship is prescribed. At greater depths still, cementation occurs and a stiffer elastic rheology becomes appropriate again. In addition, the permeability is a function $k=k(\phi)$ of porosity, with $k$ decreasing to zero fairly rapidly as $\phi$ decreases to zero.

If we suppose the basin overlies an impermeable basement at $z=0$, and its surface is at $z=h$, then suitable boundary conditions are

$$
\begin{aligned}
u^{s}=u^{l} & =0 \quad \text { at } \quad z=0, \\
\phi=\phi_{0}, \quad \dot{h} & =\dot{m}_{s}+u^{s} \quad \text { at } z=h .
\end{aligned}
$$

We suppose that $p_{e}\left(\phi_{0}\right)=0 ; \dot{m}_{s}$ is the prescribed sedimentation rate, and we take it initially to be constant.

Non-dimensionalisation and simplification of this model leads to the nonlinear diffusion equation

$$
\frac{\partial \phi}{\partial t}=\lambda \frac{\partial}{\partial z}\left\{\tilde{k}(1-\phi)^{2}\left[\frac{1}{\phi} \frac{\partial \phi}{\partial z}-1\right]\right\},
$$

wherein we make the assumptions

$$
\begin{aligned}
p_{e} & =\left[p_{e}\right]\left\{\ln \left(\phi_{0} / \phi\right)-\left(\phi_{0}-\phi\right)\right\}, \\
k & =k_{0} \tilde{k}(\phi),
\end{aligned}
$$

$\left[p_{e}\right]$ and $k_{0}$ being suitable scales for $p_{e}$ and $k$. The constitutive law for effective pressure is that of Smith (1971); it mimics the normal consolidation behaviour of compacting sediments (such as soils), and is further discussed by Audet and Fowler (1992) and Jones (1994). The dimensionless parameter $\lambda$ is given by

$$
\lambda=\frac{K_{0}}{\dot{m}_{s}},
$$

where $K_{0}=k_{0}\left(\rho_{s}-\rho_{l}\right) g / \mu$ is the surface hydraulic conductivity, and we distinguish between slow compaction $(\lambda \ll 1)$ and fast compaction $(\lambda \gg 1)$. Typical values of $\lambda$ depend primarily on the sediment type. For $\dot{m}_{s}=10^{-11}$ $\mathrm{m} \mathrm{s}^{-1}$, we have $\lambda \approx 0.1$ for the finest clay, $\lambda \approx 10^{9}$ for coarse sands. 
In general, therefore, we can expect large values of $\lambda$. The associated boundary conditions for the model become

$$
\begin{gathered}
\phi_{z}-\phi=0 \quad \text { at } z=0, \\
\phi=\phi_{0}, \quad \dot{h}=1+\lambda \tilde{k}(1-\phi)\left[\frac{1}{\phi} \frac{\partial \phi}{\partial z}-1\right] \quad \text { at } z=h .
\end{gathered}
$$

Slow compaction, $\lambda \ll \mathbf{1}$. When $\lambda$ is small, overpressuring occurs. A boundary layer analysis is easy to do, and shows that $\phi \approx \phi_{0}$ in the bulk of the (uncompacted) sediment, while a compacting boundary layer of thickness $\sqrt{\lambda t}$ exists at the base.

Fast compaction, $\lambda \gg 1$. The more realistic case of fast compaction is also the more mathematically interesting. Most simply, the solution when $\lambda \ll 1$ is the equilibrium profile

$$
\phi=\phi_{0} \exp [h-z] ;
$$

the exponential decline of porosity with depth is associated with the work of Athy (1930), but it only applies while $\lambda \tilde{k} \gg 1$. If we assume a power law for the dimensionless permeability of the form

$$
\tilde{k}=\left(\phi / \phi_{0}\right)^{8},
$$

where values of $m=8$ have been quoted (e.g. Smith 1971), then we find that $\lambda \tilde{k}$ reaches one when $\phi$ decreases to a value

$$
\phi^{*}=\phi_{0} \exp \left[-\frac{1}{m} \ln \lambda\right],
$$

and this occurs at a dimensionless depth

$$
\Pi=\frac{1}{m} \ln \lambda
$$

and time

$$
t^{*}=\frac{\Pi-\phi_{0}\left(1-e^{-\Pi}\right)}{1-\phi_{0}} .
$$

Typical values $m=8, \lambda=100, \phi_{0}=0.5$, give values $\phi^{*}=0.28, \Pi=$ $0.58, t^{*}=0.71$. In particular, a reasonable depth scale is $1 \mathrm{~km}$, and then (2.12) would correspond to a depth of $580 \mathrm{~m}$. Below this, the profile is not equilibrated, and the pore pressure is elevated. Figure 2 shows the resulting difference in the porosity profiles at $t=t^{*}$ and $t>t^{*}$, and Figure 3 shows the effect on the pore pressure, whose gradient changes abruptly from hydrostatic to lithostatic at the critical depth. 


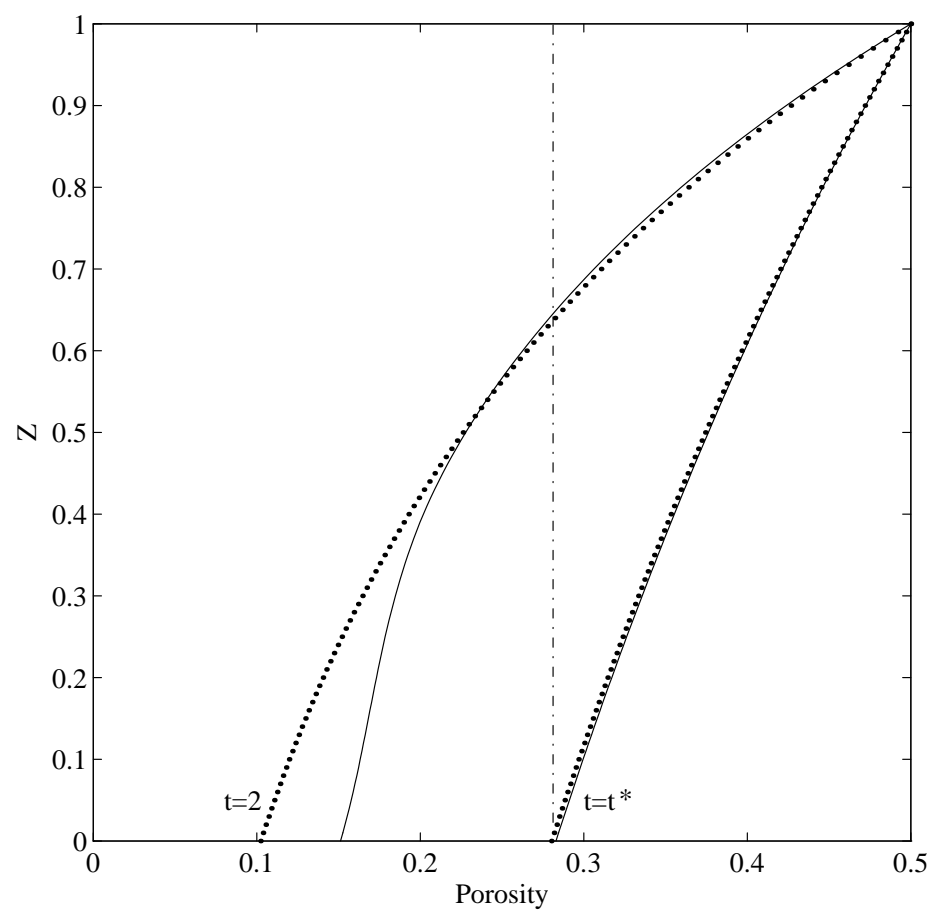

Fig. 2. Solution of (2.5) for $\lambda=100$ at times $t=t^{*} \approx 0.71$ and at $t=2$. The porosity (horizontal axis) is plotted as a function of the scaled vertical height $z / h(t)$. The solid lines are numerical solutions, whereas the dotted lines are the large $\lambda$ equilibrium profiles. There is a clear divergence at depth for $t>t^{*}$.

If we take $\phi^{*}=O(1)$ and $\lambda \gg 1$, then formally $m \gg 1$, and it is possible to analyse the profile below the critical depth. One finds that

$$
\phi=\phi^{*} \exp \left[-\frac{1}{m}\{\ln m+O(1)\}\right],
$$

which can explain the flattening evident in Figure 2, and which is also seen in field data (Freed and Peacor 1989).

Viscous compaction. These results are enlightening, but perhaps are based on an incorrect rheology. Below a depth of perhaps a kilometre, pressure solution at intergranular contacts becomes important, and the resulting dissolution and local reprecipitation leads to an effective creep of the grains (and hence of the bulk medium) in a manner analogous to regelation in ice. Early work on the subject was by Angevine and Turcotte (1983) and Birchwood and Turcotte (1984). More recently, Fowler and 


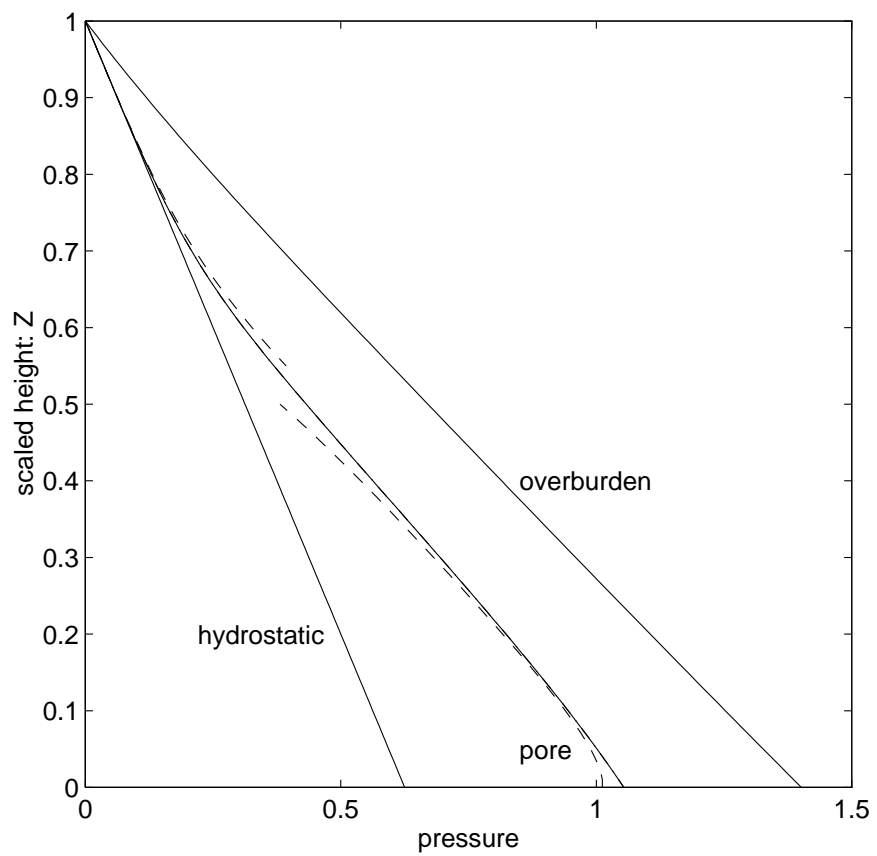

FIG. 3. Hydrostatic, overburden (lithostatic) and pore pressures at $t=5$ and $\lambda=$ 100 , as functions of the scaled height $z / h(t)$. The transition from equilibrium to nonequilibrium compaction at the critical depth is associated with a transition from normal to abnormal pore pressures. The dashed lines represent two distinct approximations to the pore pressure profile, respectively valid above and below the transition region. Further details are given by Fowler and Yang (1998).

Yang (1999) showed that for such viscous compaction, the constitutive relation for the effective pressure becomes

$$
p_{e}=-\xi \nabla \cdot \mathbf{u}^{s} .
$$

In one dimension, the resulting dimensionless model is

$$
\begin{aligned}
& -\frac{\partial \phi}{\partial t}+\frac{\partial}{\partial z}[(1-\phi) u]=0, \\
& u=-\lambda \tilde{k}\left[\frac{\partial p}{\partial z}+1-\phi\right], \\
& p=-\Xi \frac{\partial u}{\partial z}
\end{aligned}
$$

where $p$ is the scaled effective pressure. The compaction parameter is the same as before, and the extra parameter $\Xi$ can be taken to be of $O(1)$ for typical basin depths of kilometres. Boundary conditions for (2.16) are 


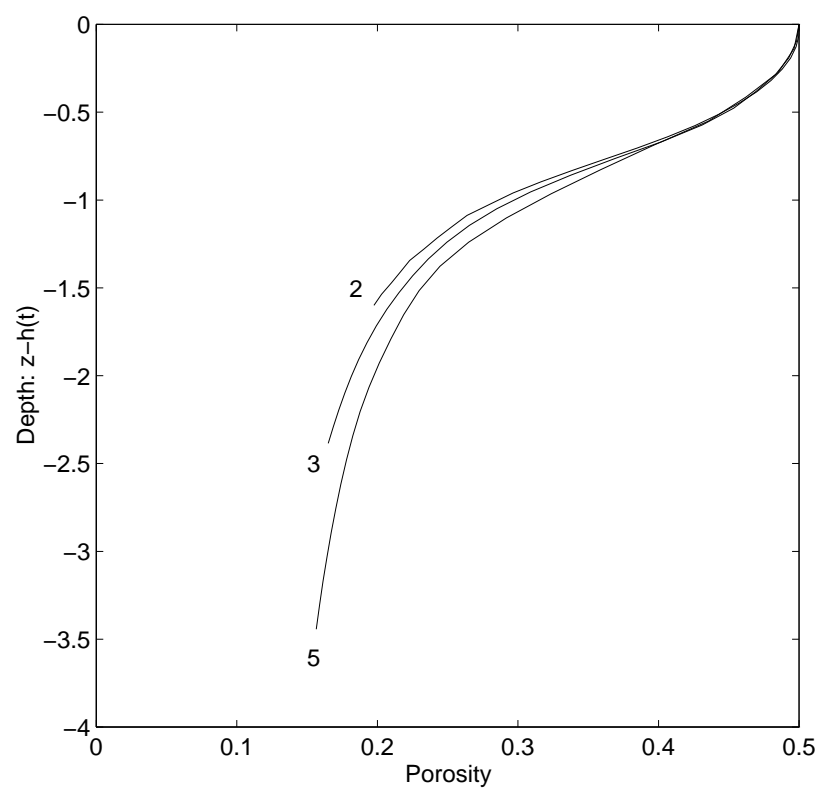

FIG. 4. Evolution of the porosity as a function of depth $h-z$, with a viscous rheology, at $\lambda=100$. The upper concave part is in equilibrium, while overpressuring occurs where the profile is flatter below this.

$$
\begin{aligned}
& u=0 \quad \text { on } z=0, \\
& p=0, \quad \phi=\phi_{0}, \quad \dot{h}=1+u \quad \text { at } z=h .
\end{aligned}
$$

This system can also be studied asymptotically. When $\lambda \ll 1$, compaction is slow and a basal compaction layer again forms. When $\lambda \gg 1$, explicit solutions can again be obtained. There is an upper layer at equilibrium, but now the porosity decreases concavely with depth. As before, there is a transition when $\phi=\phi^{*}$, and below this

$$
\phi=\phi^{*} \exp \left[-\frac{2}{m}\{\ln m+O(1)\}\right],
$$

similar to (2.14).

The main distinction between viscous and elastic compaction is thus in the form of the rapidly compacted equilibrium profile near the surface (Figure 4). The concave profile is not consistent with observations, but we need not expect it to be, as the viscous behaviour of pressure solution only cuts in at reasonable depths. A more general relation which allows for this is a viscoelastic compaction law of the form

$$
\boldsymbol{\nabla} \cdot \mathbf{u}^{s}=-\frac{1}{K_{e}} \frac{d p_{e}}{d t_{s}}-\frac{p_{e}}{\xi}
$$

and this has been studied by Yang (2000). 


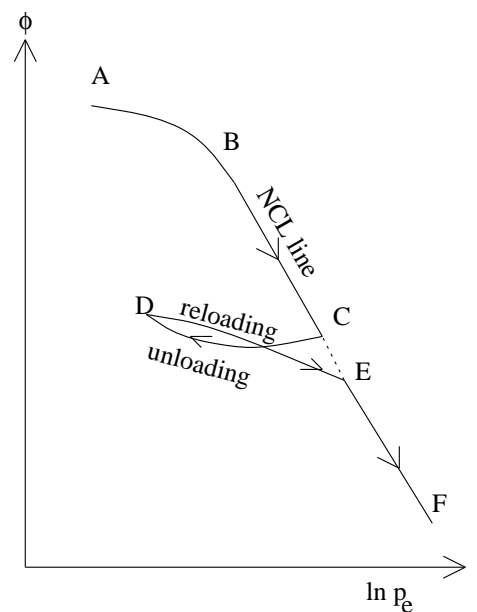

(a)

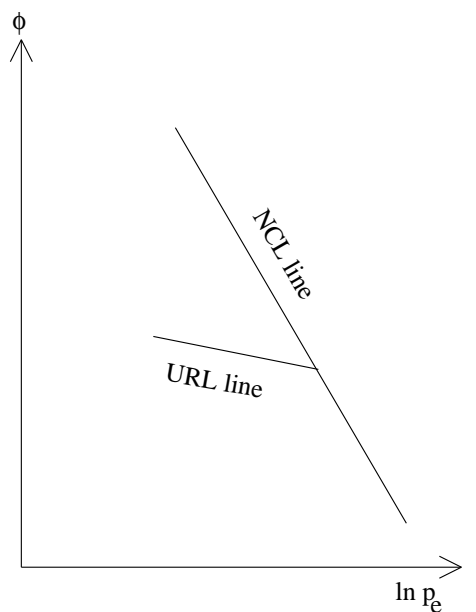

(b)

FIG. 5. Non-linear behaviour of soil consolidation: (a) typical experimental result for the normal consolidation line (NCL), and the hysteretic effect of unloading and reloading; (b) corresponding idealised model of loading/unloading: URL is the unloading and reloading line.

Hysteresis. Like soils, it is natural to expect that compaction in sediments is not reversible, so that the elastic compaction law $p_{e}(\phi)$ should be modified as indicated in Figure 5. In keeping with (2.6), one convenient choice of constitutive relation is the following. We define

$$
\begin{aligned}
& \phi_{0}^{*}(z, t)=\min _{\tau} \phi(z, \tau<t), \\
& \tilde{p}^{*}(z, t)=\max _{\tau} \tilde{p}(z, \tau<t),
\end{aligned}
$$

where the scaled maximum effective pressure $\tilde{p}^{*}$ is given by

$$
\tilde{p}^{*}=\ln \left(\phi_{0} / \phi_{0}^{*}\right)-\left(\phi_{0}-\phi_{0}^{*}\right) .
$$

On the normal consolidation line (NCL), we have

$$
\tilde{p}=\ln \left(\phi_{0} / \phi\right)-\left(\phi_{0}-\phi\right)
$$

if $\tilde{p}=\tilde{p}^{*}$ and $\partial \phi / \partial t \leq 0$. Otherwise we are on the unloading-reloading line (URL), which we define to be

$$
\tilde{p}=\left[1-(1-\gamma) \phi_{0}^{*}\right] \ln \left[\frac{\phi_{0}^{*} \gamma}{\phi-(1-\gamma) \phi_{0}^{*}}\right]-\left(\phi_{0}^{*}-\phi\right)+\tilde{p}^{*} .
$$

An illustration of this choice is given in Figure 6, in the usual soil consolidation variables of void ratio $\phi /(1-\phi)$ versus $\ln p_{e}$ (or $\left.\ln \tilde{p}\right)$, for the choice $\gamma=0.2$ (the particular URL shown is for $\phi_{0}^{*}=0.3$ ). 


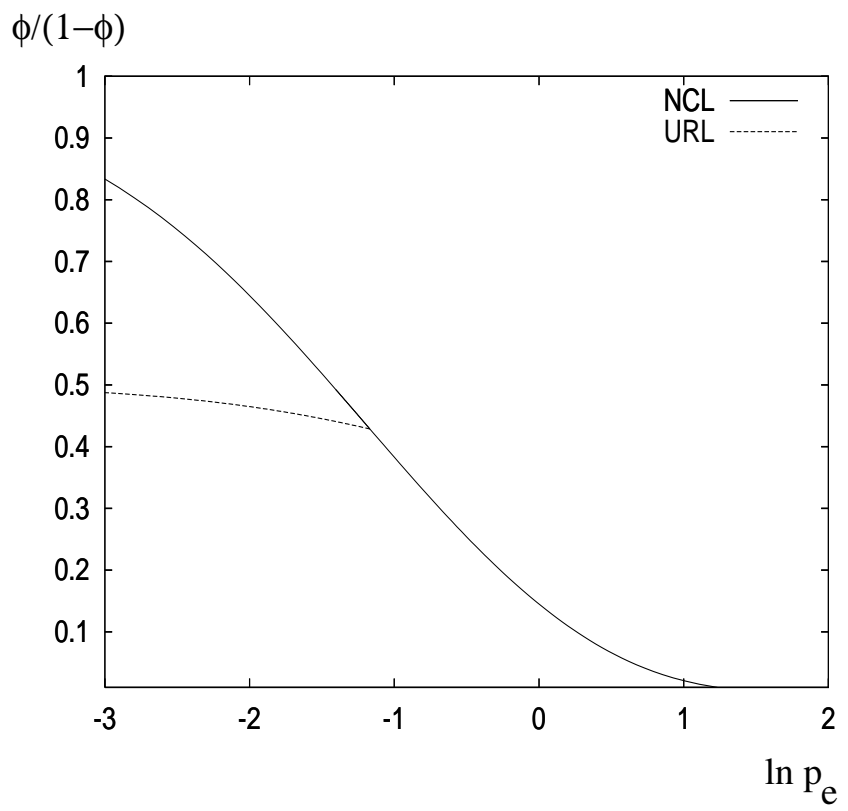

FIG. 6. Normal consolidation line and one particular unloading/reloading line for (2.23), using $\gamma=1$ and $\gamma=0.2$.

Two effects of hysteresis when $\lambda \gg 1$ are seen in Figures 7 and 8 . In Figure 7 an equilibrated porosity profile is subjected to unloading via denudation at the top. An apparent interface moves downward, the depth $s(t)$ of which is initially well approximated by the formula (Fowler and Yang 2000a)

$$
s(t)=\frac{1}{m-1} \ln \left[\frac{\lambda(m-1)^{2}\left(1-\phi_{0}\right)^{2}}{\gamma \phi_{0}} t+1\right],
$$

which can be obtained on the basis that $m$ is large.

In Figure 8, it can be seen that successive cycles of loading and unloading lead to a discontinuity in the porosity when the unloaded top part of the basin is reloaded. On the other hand, unloading of a loaded region leads to a jump in the porosity gradient. These discontinuities eventually disppear with time.

3. Diagenesis. Diagenesis refers generally to the process of chemical alteration to rock, and here we specifically intend it to mean the conversion of smectite to illite via a dewatering reaction. The resultant release of water is a potential cause of excess pore pressures, but importantly in the present context, we show how use of a weak solubility limit allows enormous simplification of quite complicated reaction schemes. 
(a)

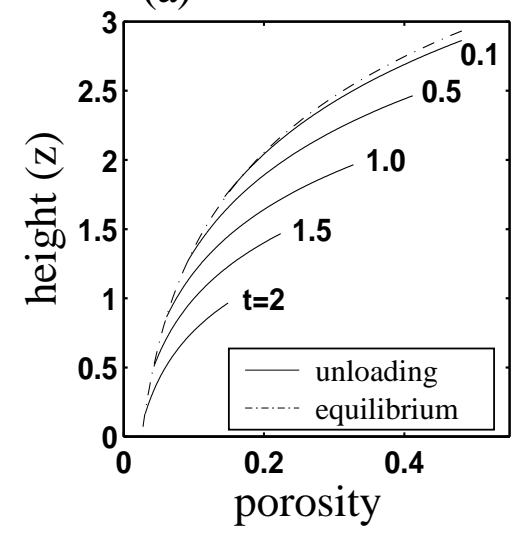

(b)

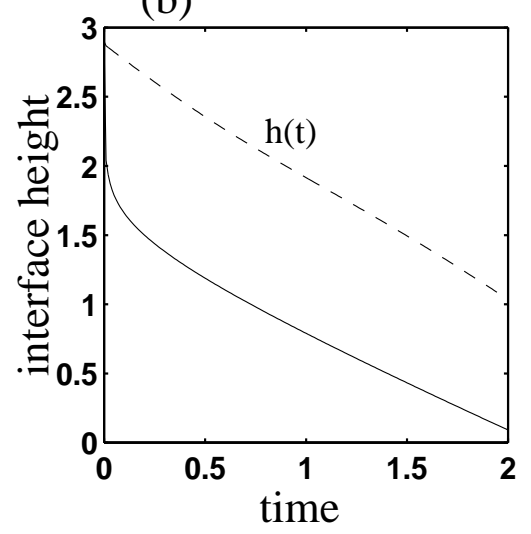

FIG. 7. The left hand figure shows the result of unloading an equilibrated exponential porosity profile, using the hysteretic rheology, with $\lambda=1500$ and $\gamma=0.25$. An apparent interface moves downwards, and the right hand figure shows its position as a function of time (solid line) together with the position of the top surface (dashed line).
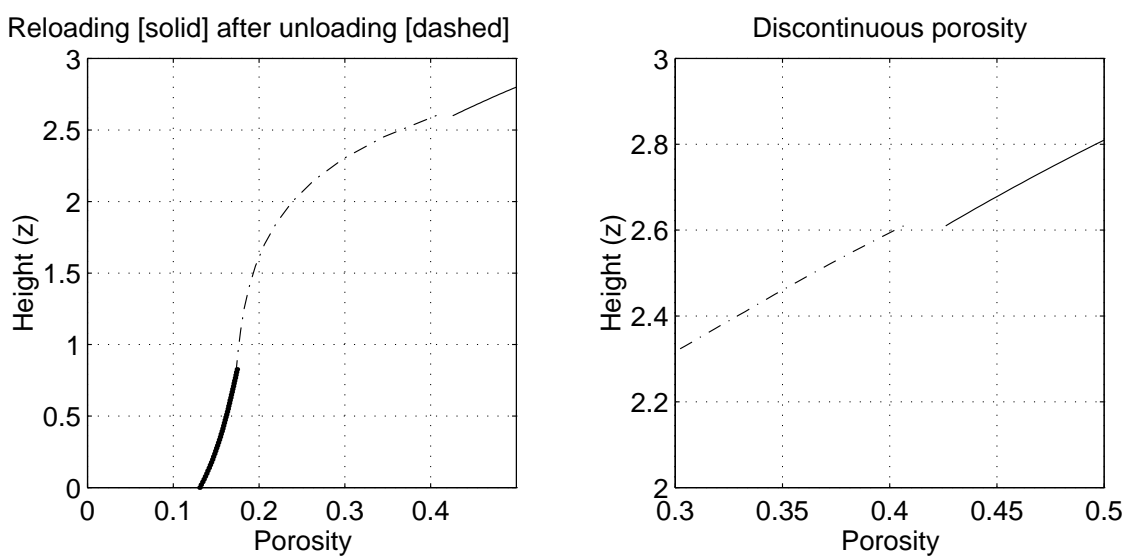

FIG. 8. Cyclic loading and unloading. The left hand figure shows a snapshot of the porosity profile $(\lambda=100, \gamma=0.2)$; the regions correspond to reloading, unloading, loading, from the top down. At the upper junction, it can be seen that there is a porosity discontinuity, while at the lower junction there is a slight but definite jump in the porosity gradient. The right hand figure shows an enlargement of the upper discontinuity.

One view of the smectite-illite reaction is to treat it using first order kinetics, thus

$$
\mathrm{S}^{S} \rightarrow \mathrm{I}^{S}+n \mathrm{H}_{2} \mathrm{O}
$$


where each mole conversion yields $n$ moles of water. Such a scheme is not inconsistent with at least some experimental data (Eberl and Hower 1976), and the rate constant involved has an activation energy in the range 60-80 $\mathrm{kJ} \mathrm{mol}{ }^{-1}$.

However, it is likely that the transformation of smectite to illite occurs through a compound sequence of precipitation and dissolution, perhaps the simplest such being the following:

$$
\begin{gathered}
\mathrm{S}^{S} \stackrel{R_{1}}{\longrightarrow} \mathrm{X}^{L}+n \mathrm{H}_{2} \mathrm{O}, \\
\mathrm{KFs} \stackrel{R_{2}}{\longrightarrow} \mathrm{K}^{+L}+\mathrm{Al}(\mathrm{OH})_{4}^{-L}+s \mathrm{SiO}_{2}^{L}, \\
\mathrm{~K}^{+L}+\mathrm{Al}(\mathrm{OH})_{4}^{-L}+f \mathrm{X}^{L} \stackrel{R_{3}}{\longrightarrow} f \mathrm{I}^{S}+\mathrm{SiO}_{2}^{L}, \\
\mathrm{SiO}_{2}^{L} \underset{R_{4}^{-}}{\stackrel{R_{4}^{+}}{\rightleftharpoons}} \mathrm{Qz} .
\end{gathered}
$$

The smectite ( $\mathrm{S}^{S}$, the superscript denotes the solid phase) dissolves to form an aqueous silica combination $\mathrm{X}^{L}$, such as $\mathrm{Si}_{4} \mathrm{O}_{10}(\mathrm{OH})_{2}$. Additionally, illite precipitation requires potassium ions, and these may be obtained from the dissolution of potassium feldspar (in the second reaction); the aluminium hydroxyl ions $\mathrm{Al}(\mathrm{OH})_{4}^{-L}$ act in the same way. The silica combination now combines with the potassium and aluminium to form illite precipitate, together with aqueous silica $\mathrm{SiO}_{2}^{L}$, which itself precipitates as quartz Qz. Taking suitable multiples of the reactions and adding to eliminate the aqueous phases, the overall reaction is found to be

$$
\mathrm{S}+f^{-1} \mathrm{KFs} \stackrel{R}{\longrightarrow} \mathrm{I}+n \mathrm{H}_{2} \mathrm{O}+f^{-1}(s+1) \mathrm{Qz} .
$$

Ideally we would like to be able to write kinetics for (3.3) analogously to (3.1), with a recipe for the effective reaction rate $R$.

It turns out, at least for this reaction scheme (but we might suspect more generally), that the use of the weak solubility limit (or solid density asymptotics in Ortoleva's (1994) terms) allows such a recipe to be found. To illustrate the method, note that conservation equations for the concentrations $S, X, F, K, A, L, Q, I$ of the substances smectite, aqueous silica combination, feldspar, potassium ions, aluminium hydroxyl ions, quartz and illite satisfy equations of the type

$$
\begin{aligned}
\frac{\partial}{\partial t}[(1-\phi) S]+\nabla \cdot\left[(1-\phi) S \mathbf{u}^{s}\right] & =-r_{1}, \\
\frac{\partial}{\partial t}(\phi X)+\nabla \cdot\left(\phi X \mathbf{u}^{l}\right)-\nabla \cdot(\phi D \nabla X) & =r_{1}-f r_{3},
\end{aligned}
$$

and so on, where the reactions rates $r_{i}$ are scaled with respect to the surface rates $R_{i}$ by the specific interfacial surface areas $\Sigma_{i}$ (thus $r_{i}=\Sigma_{i} R_{i}$; details 
can be found in Fowler and Yang (2000b)). There are six other equations of this type, together with a water conservation equation (this is the equation for $\phi$ ). Of the total of nine equations, four are for aqueous concentrations. That for $A$ is identical to that for $K$, and we ignore it henceforth.

The weak solubility limit is associated with the observations that aqueous dissolved species are typically present in trace quantities of the order of $10-100 \mathrm{ppm}\left(1 \mathrm{ppm}=10^{-3} \mathrm{~kg} \mathrm{~m}^{-3}\right)$, and thus the concentrations of the aqueous phases such as $X$ are much less than those of the solid phases, such as $S$. When the model is suitably non dimensionalised, the result is that the transport terms for the aqueous phases are very small, so that the corresponding reaction terms can be taken to be in equilibrium. From this, we obtain the three relationships

$$
\begin{aligned}
r_{1} & \approx f r_{3}, \\
r_{2} & \approx r_{3}, \\
r_{4}^{+}-r_{4}^{-} & \approx(s+1) r_{3} .
\end{aligned}
$$

Since the rate of dissolution of $S$ is $r_{1}$ and the rate of precipitation of $I$ is $f r_{3}$, this immediately shows that first order kinetics of the form (3.1) does apply, with the reaction rate $f r_{3}$. Since $r_{4}^{+}$is a precipitation rate, and $r_{4}^{-}$the dissolution rate of the same mineral pair $\mathrm{SiO}_{2}^{L} \leftrightarrow \mathrm{Qz}$, and since either $r_{4}^{+}=0$ or $r_{4}^{-}=0$, it is apparent that $(3.5)_{3}$ determines $r_{4}^{+}$and $r_{4}^{-}$ together. Thus all the reaction rates can be written in terms of $r_{3}$, and this then determines the aqueous phase pseudo-equilibrium concentrations of $X, L$ and $K$ in terms of $r_{3}$ and various temperature dependent rate factors, since the kinetic rates $r_{i}$ are prescribed in terms of these.

If the smectite equation (3.4) $)_{1}$ is written in terms of smectite volume fraction $\phi_{S}$, then it becomes

$$
\frac{\partial \phi_{S}}{\partial t}+\nabla \cdot\left[\phi_{S} \mathbf{u}^{s}\right]=-\frac{M_{S}}{\rho_{S}} f r_{3},
$$

where $M_{S}$ is the molecular weight of smectite, and $\rho_{S}$ its density, and there are three similar equations for $\phi_{F}, \phi_{Q}, \phi_{I}$ (all the right hand sides being proportional to $r_{3}$ ). In addition, the porosity $\phi$ satisfies

$$
\frac{\partial \phi}{\partial t}+\nabla \cdot\left(\phi \mathbf{u}^{l}\right)=n \frac{M_{w}}{\rho_{w}} f r_{3},
$$

The illite precipitation rate is given in terms of the aqueous phase (supersaturated) concentrations of $K$ and $X$ (ignoring $A$, or lumping it with $K$ ), which are themselves given in terms of $r_{3}$; we thus finally determine the effective first order kinetic rate constant.

In the present case, the dimensionless version of (3.6) can be written as

$$
\frac{\partial \phi_{S}}{\partial t}+\nabla \cdot\left[\phi_{S} \mathbf{u}^{s}\right]=-R^{*},
$$


with a corresponding equation for $\phi$, and the expression for $R^{*}$ is

$$
R^{*} \approx f \phi_{I} \mathcal{R}_{I P}\left[1-\frac{R^{*}}{\phi_{S} \mathcal{R}_{S D}}\right]\left[1-\frac{R^{*}}{\phi_{F} \mathcal{R}_{F D}\left\{f\left(\theta_{L}-1\right)-\frac{(s+1) R^{*}}{\phi_{Q} \mathcal{R}_{Q P}}\right\}}\right]
$$

In this expression $\mathcal{R}_{I P}, \mathcal{R}_{S D}, \mathcal{R}_{F D}$ and $\mathcal{R}_{Q P}$ are temperature dependent rate factors for illite precipitation, smectite dissolution, feldspar dissolution and quartz precipitation. The constant $\theta_{L}$ is the solubility ratio $c_{L F} / c_{L Q}$, where $c_{L F}$ is the solubility limit of $L$ in the presence of feldspar, and $c_{L Q}$ is the solubility limit of $L$ in the presence of quartz; it is of $O(1)$, whereas equivalent other solubility ratios $\theta_{K}=c_{K I} / c_{K F}$ and $\theta_{X}=c_{X I} / c_{X S}$ have been taken as small.

A general complication in solving for $\phi_{S}$ is that $R^{*}$ depends on $\phi_{S}, \phi_{I}$, $\phi_{Q}$ and $\phi_{F}$. Given $\mathbf{u}^{s},(3.8)$ is hyperbolic for $\phi_{S}$ with boundary condition $\phi_{S}=\phi_{S}^{0}$ on the upper surface $z=h$. The equations for the solid fractions $\phi_{Y}, Y=I, Q$ and $F$, are all of the form

$$
\frac{\partial \phi_{Y}}{\partial t}+\nabla \cdot\left[\phi_{Y} \mathbf{u}^{s}\right]=\alpha_{Y} R^{*}
$$

and if $\phi_{Y}=\phi_{Y}^{0}$ on $z=h$, then $\phi_{Y}$ can be written as a linear combination of $\phi_{S}$ and $\phi_{I}$

$$
\phi_{Y}=\frac{\left(\alpha_{Y} \phi_{S}^{0}+\phi_{Y}^{0}\right) \phi_{I}+\left(\alpha_{I} \phi_{Y}^{0}-\alpha_{Y} \phi_{I}^{0}\right) \phi_{S}}{\alpha_{I} \phi_{S}^{0}+\phi_{I}^{0}} .
$$

Therefore, the reaction rate $R^{*}$ can generally be written explicitly as a function of $\phi_{S}$ and $\phi_{I}$, and the diagenesis model collapses to equations for $\phi_{S}, \phi_{I}$ and $\phi$, together Darcy's law.

In one space dimension, we find

$$
\begin{gathered}
\frac{\partial \phi_{S}}{\partial t}+\frac{\partial}{\partial z}\left(\phi_{S} u^{s}\right)=-R^{*} \\
\frac{\partial \phi_{I}}{\partial t}+\frac{\partial}{\partial z}\left(\phi_{I} u^{s}\right)=\alpha_{I} R^{*} \\
\frac{\partial \phi}{\partial t}=\frac{\partial}{\partial z}\left[(1-\phi) u^{s}\right]+\left(\alpha+\alpha_{w}\right) R^{*} \\
u^{s}=-\alpha \psi-\frac{\lambda(1-\phi) \tilde{k}}{\phi}\left(\frac{\partial \phi}{\partial z}-\phi\right),
\end{gathered}
$$

where

$$
\psi=\int_{0}^{z} R^{*} d z
$$



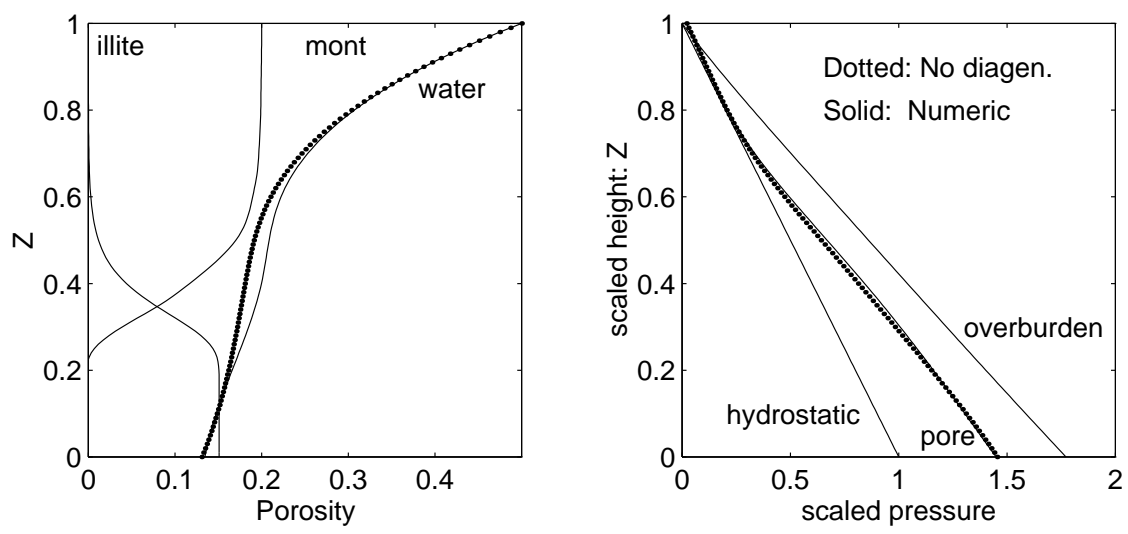

FIG. 9. On the left, vertical profiles (with scaled height $z / h(t)$ ) of smectite, illite and water porosities, at $t=5$ with $\lambda=100$. Of the two water curves, the dotted one is the profile without diagenesis, and the effect of diagenesis is small. On the right, the effect on overpressuring is minor. The diagenetic window typically lies below the transition to non-equilibrium compaction.

The equation for $\phi$ is parabolic as before, and the equations for $\phi_{S}$ and $\phi_{I}$ are hyperbolic, the characteristics propagating downwards from the top surface.

When $\lambda \ll 1$ (slow compaction), diagenesis causes negative pore pressures to occur. In reality, fracturing would occur, and this may be one mechanism whereby joints form in fine-grained sedimentary rocks. When $\lambda \gg 1$, numerical solutions (see Figure 9) indicate that pore water release is not significant in raising pore pressure, essentially because the parameter $\alpha_{I}=0.86$ in (3.12) is close to one. The other observation (which can also be predicted analytically) is that the diagenetic reaction window lies below the transition from equilibrium compaction $\left(\phi>\phi^{*}\right)$ to non-equilibrium compaction $\left(\phi<\phi^{*}\right)$.

4. Discussion. Compaction in sedimentary basins is essentially similar to consolidation in groundwater flow, and is described by a nonlinear diffusion equation for the porosity. One principal dimensionless parameter $(\lambda)$ describes the process, this being the ratio of the hydraulic conductivity to the sedimentation rate, and it is possible to analyse the solutions in the limits of fast $(\lambda \gg 1)$ and slow $(\lambda \ll 1)$ compaction.

Interest in compaction is motivated by its occurrence in sedimentary basins, and also by issues of subsidence due to groundwater or natural gas extraction (see, for example, Baú et al. 2000). We have shown how compaction can explain overpressuring at depth due to the reduction of permeability as the porosity decreases. In addition, we can include complicating effects such as hysteresis and diagenesis, and these incorporate interesting mathematical as well as physical effects. Hysteresis in sediment compaction 
leads to a "diffusion" equation for $\phi$ where the diffusion coefficient depends on past history. In conditions of cyclic loading and unloading, jumps in porosity gradient can occur and, more surprisingly, jumps on porosity also occur.

Diagenesis has a relatively minor quantitative effect on overpressuring. What is perhaps of more interest is that a fairly complicated sequence of precipitation/dissolution steps can be reduced, in the limit of weak solubility, to a model with first order kinetics, albeit with a complicated (but explicitly defined) reaction rate. In fact, this observation is likely to be true in general. Suppose we have a sequence of precipitation and dissolution steps for solids $\mathrm{S}_{i}$ and liquids $\mathrm{L}_{j}$ :

$$
\begin{aligned}
& \mathrm{L}_{1}+\ldots \stackrel{R_{1}}{\longrightarrow} \mathrm{S}_{1}+\ldots, \\
& \mathrm{S}_{2}+\ldots \stackrel{R_{2}}{\longrightarrow} \mathrm{L}_{2}+\ldots
\end{aligned}
$$

Each reaction step necessarily involves at least one aqueous phase component, and thus all the reaction rates $R_{1}, \ldots, R_{n}$ occur in the conservation equations for the aqueous phase components. Since these can all be taken to be in equilibrium, then if there are $k$ different aqueous phase components, we obtain $k$ relations for the $n$ reactions. If $k=n-1$, then all the reaction rates can be written in terms of the overall production rate, and first order kinetics will apply.

In the present example (3.2), there are five reaction steps, and three aqueous components (lumping $\mathrm{K}^{+L}$ and $\mathrm{Al}(\mathrm{OH})_{4}^{-L}$ together), but the precipitation/dissolution of quartz is effectively one reaction (either but not both at once can occur), and so the condition $n=k+1$ is effectively met. More generally, we see that the production of solid precipitate $P$ from solid substrates $S$ through a sequence of intermediate dissolution/precipitation steps may often lead to this situation.

Seals. One process which we have not described is the formation of high pressure seals. In certain circumstances, pore pressures undergo fairly rapid jumps across a 'seal', typically at depths of $3000 \mathrm{~m}$. Such jumps cannot be predicted within the confines of a simple compaction theory, and require a mechanism for pore-blocking. Mineralisation is one such mechanism, as some seals are found to be mineralised with calcite and silica (Hunt 1990). In fact, a generalisation of the clay diagenesis model to allow for calcite precipitation could be used for this purpose. As it stands, $(3.12)_{3}$ predicts a source for $\phi$, but mineralisation would cause a corresponding sink term. Reduction of $\phi$ leads to reduction of diffusive transport, and the feedback is self-promoting. Problems of this type have been studied by Ortoleva (1994), for example.

Acknowledgement. I am grateful to the IMA and its Director, Willard Miller, for the invitation to spend two weeks at the IMA in January, 2000. I am also extremely grateful to all the staff of the IMA who 
were uniformly helpful and friendly, and who are instrumental in providing a very pleasant working environment.

\section{REFERENCES}

Abercrombie, H.J., I.E. Hutcheon, J.D. Bloch, And P. De Caritat, 1994. Silica activity and the smectite-illite reaction, Geology 22, 539-542.

Angevine, C.L. And D.L. TurcotTe, 1983. Porosity reduction by pressure solution: a theoretical model for quartz arenites. Geol. Soc. Amer. Bull. 94, 1129-1134.

Атну, L.F., 1930. Density, porosity, and compaction of sedimentary rocks. Amer. Assoc. Petrol. Geol. Bull. 14, 1-22.

Audet, D.M. AND A.C. Fowler, 1992. A mathematical model for compaction in sedimentary basins, Geophys. J. Int. 110, 577-590.

Baú, D., G. Gambolati AND P. Teatini, 2000. Residual land subsidence near abandoned gas fields raises concern over Northern Adriatic coastland. EOS 81, no. 22, pp. $245,248-9$.

Birchwood, R.A. AND D.L. TurcotTe, 1994. A unified approach to geopressuring, low-permeability zone formation, and secondary porosity generation in sedimentary basins. J. Geophys. Res. 99, 20, 051-20, 058.

Dewynne, J.N., A.C. Fowler And P.S. Hagan, 1993. Multiple reaction fronts in the oxidation-reduction of iron-rich uranium ores. SIAM J. Appl. Math. 53, 971-989.

EBerL, D. AND J. HowER, 1976. Kinetics of illite formation. Geol. Soc. Amer. Bull. $\mathbf{8 7}, 1326-1330$.

FOWLER, A.C., 1985. A mathematical model of magma transport in the asthenosphere. Geophys. Astrophys. Fluid Dyn. 33, 63-96.

Fowler, A.C. AND X.S. YANG, 1998. Fast and slow compaction in sedimentary basins. SIAM J. Appl. Math. 59, 365-385.

FOWLER, A.C. AND X.S. YANG, 1999. Pressure solution and viscous compaction in sedimentary basins. J. Geophys. Res. 104, 12, 989-12, 997.

FOWLER, A.C. AND X.S. YANG, 2000a. Loading and unloading of sedimentary basins: the effect of rheological hysteresis. J. Geophys. Res., submitted.

FOwLER, A.C. AND X.S. YANG, 2000b. Precipitation-dissolution mechanisms for diagenesis in sedimentary basins. J. Geophys. Res., submitted.

Freed, R.L. AND D.R. PeAcor, 1989. Geopressured shale and sealing effect of smectite to illite transition. AAPG Bull. 73, 1223-1232.

HunT, J.M., 1990. Generation and migration of petroleum from abnormally pressured fluid compartments. AAPG Bull. 74, 1-12.

Jones, M., 1994. Mechanical principles of sediment deformation. In: The geological deformation of sediments, ed. A. Maltman, pp. 37-71, Chapman and Hall, London.

McKenzie, D.P., 1984. The generation and compaction of partial melts. J. Petrol. 25, $713-765$.

Ortoleva, P., E. Merino, C. Morse AND J. Chadam, 1987. Geochemical selforganisation I: Reaction-transport feedbacks and modeling approach. Amer. J. Sci. 287, 979-1, 007.

Ortoleva, P., 1994. Geochemical self-organisation. O. U. P., Oxford.

ScotT, D.R. And D.J. Stevenson, 1984. Magma solitons. Geophys. Res. Letts. 11, 1, $161-1,164$.

Sмiтh, J.E., 1971. The dynamics of shale compaction and evolution in pore-fluid pressures. Math. Geol. 3, 239-263.

YANG, X.S., 2000. Nonlinear viscoelastic compaction in sedimentary basins. Nonlin. Process. Geophys. 7, 1-7. 